\title{
Las asignaturas de salud pública en las políticas de formación para la carrera de medicina
}

\section{Public health subjects present in the formative policies of the medical career}

\author{
Dr. J uan Vela Valdés,' Dr. J osé Fernández Sacasas" \\ I Escuela Nacional de Salud Pública. La Habana, Cuba. \\ "Universidad de Ciencias Médicas de La Habana. La Habana, Cuba.
}

\section{RESUMEN}

I ntroducción: en 1994 se realizó una investigación por encargo de la OPS, acerca de las disciplinas de salud pública en el plan de estudios de la carrera de medicina en Cuba. En esa investigación se identificaron un conjunto de problemas y se hicieron recomendaciones.

Objetivos: dar respuesta a los problemas y recomendaciones detectadas en la investigación de 1994 y brindar información relevante a los claustros y directivos académicos acerca del plan de estudio perfeccionado de la carrera de medicina elaborado en el 2010.

Métodos: se realizó un estudio cualitativo con el objetivo de comparar el grado de participación curricular del conjunto de las asignaturas de salud pública en el plan de estudio perfeccionado en el 2010 de la carrera de medicina a partir del programa vigente en 1985 . Se tomaron los resultados de la investigación realizada en 1994 en el Instituto Superior de Ciencias Médicas de La Habana y el plan de estudio de medicina perfeccionado del 2010. A la información recopilada se le aplicó análisis de concordancia y diferencia.

Resultados: se introdujeron cambios en las formas organizativas de la enseñanza, incremento de contenidos, aumento de las horas de la educación en el trabajo y de horas prácticas, entre otros. La asignatura de medicina general integral fue la disciplina integradora de todo el currículo.

Conclusiones: las limitaciones identificadas en la investigación de 1994 se eliminaron en el perfeccionamiento del plan de estudio de 2010. Es necesario que los resultados de esta investigación se tengan en cuenta en las futuras evaluaciones curriculares de la carrera de medicina.

Palabras clave: salud pública, política formativa, planes de estudio, evaluación, Cuba. 


\section{ABSTRACT}

Introduction: at the request of PAHO, a research study was conducted in 1994 on the public health disciplines included in the medical career curriculum in Cuba. A number of problems that had to be addressed and of recommendations that had to be improved were then identified.

Objectives: to respond to these problems and recommendations so as to provide relevant information to the faculties and to academic managers on the upgraded curriculum of the medical career in 2010.

Methods: a qualitative study was made to compare the level of participation of the set of public health subjects in the upgraded curriculum of the medical studies in 2010, taking the 1985 program as a basis. The results of the 1994 research study at the Higher Institute of Medical Sciences of Havana and the 2010 upgraded medical curriculum were both considered. Concordance and difference analysis techniques were applied to the gathered information.

Results: the organization patterns of teaching were changed by increasing the number of contents, the number of hours devoted to education at work and of practice hours, among others. Integrated general medicine was the agglutinating discipline in the whole curriculum.

Conclusions: the identified restrictions in the 1994 research work are eliminate in the improved 2010 curriculum. It is required that the results of this research study be taken into account in the curricular evaluations of the medical career in the future.

Key words: public health, formative policy, curricula, evaluation, Cuba.

\section{NTRODUCCI ÓN}

El estudio de la enseñanza de las disciplinas de Salud Pública en el plan de estudio de la carrera de medicina perfeccionado en 2010, se justifica por la innegable importancia que le aportan a la formación del médico. En Cuba, el plan de estudio de esta carrera ha tenido diferentes cambios para su perfeccionamiento continuo de acuerdo a los requerimientos provenientes del sistema de salud, o en busca de un mejor desarrollo del proceso docente educativo, en respuesta a los avances científicos del mundo y las tendencias de la educación médica, fundamentalmente con la orientación a la Atención Primaria de Salud (APS). Las disciplinas de Salud Pública no se escapan de este perfeccionamiento.

En el año 1994 se realizó una investigación por encargo de la Organización Panamericana de la Salud (OPS), como parte de una investigación regional, acerca de las disciplinas de Salud Pública en el plan de estudios de medicina de Cuba. ${ }^{1}$

En esa investigación se identificaron un conjunto de problemas y recomendaciones con el fin de perfeccionar estas disciplinas en el programa. En el curso escolar 2010-2011, después de un año de trabajo intenso de la Universidad de Ciencias Médicas de La Habana (UCMH), con la participación de los profesores de todas las universidades médicas y de la Dirección de Docencia del MINSAP, se pone en vigor el plan de estudios de Medicina perfeccionado. Por tal motivo nos preguntamos: 
¿Qué transformaciones han sufrido las disciplinas de Salud Pública en el plan de estudios perfeccionado de la carrera de medicina en el 2010?

¿Se tomaron en cuenta las recomendaciones del estudio realizado en 1994 para el perfeccionamiento de las disciplinas de Salud Pública?

El presente estudio se propuso dar respuesta a estas interrogantes con la finalidad de brindar información relevante a los claustros y directivos académicos acerca del plan de estudio perfeccionado de la carrera de medicina elaborado por la Comisión Nacional de Carrera de medicina de la UCMH, como centro rector, en el año 2010.

\section{MÉTODOS}

Se realizó un estudio cualitativo con el objetivo de comparar el grado de participación curricular del conjunto de las asignaturas de salud pública en el plan de estudio perfeccionado en el 2010 de la carrera de medicina a partir del programa vigente en 1985. Se tomaron los resultados de la investigación realizada en 1994 en el Instituto Superior de Ciencias Médicas de La Habana (ISCM H) y el plan de estudio de medicina perfeccionado del 2010. A la información recopilada se le aplicó análisis de concordancia y diferencia.

\section{RESULTADOS}

\section{Las disciplinas de Salud Pública en el plan de 1994}

El plan de estudios de Medicina en 1994 contaba con 7841 h totales, con el 21,2\% en teoría, 20,3 en práctica y 58,5\% en Educación en el Trabajo. Las asignaturas de Salud Pública alcanzaban un fondo de tiempo de 1342 h, lo que significa el $17 \%$ de las horas totales (tabla 1 ).

El plan de estudio de la carrera de medicina de 1994 en sus bases teóricas, en los contenidos formas y métodos de enseñanza, así como en su diseño, responde a las necesidades de salud de la sociedad cubana actual y a su futuro nivel de desarrollo. Las perspectivas de las disciplinas de Salud Pública en el plan de estudio, tienden a aumentar las coordinaciones entre ellas en una primera etapa y encaminarse a la integración a un mediano plazo. Estas disciplinas, en contacto tan directo con nuestra sociedad y con el sistema de salud, reciben el impacto de los cambios que en los mismos sistemáticamente se producen, por lo que sus programas deben estar en un perfeccionamiento continuo y abordarse de forma conjunta docencia- asistencia. Un objetivo a lograr es ampliar aún más el espacio de la APS en el proceso de formación, de manera que llegue a permear todo el plan de estudio de 1ro. al 6to. año. Para cumplir este objetivo se debe continuar mejorando la calidad y eficiencia de los servicios de salud en la APS que posibiliten el aseguramiento del aprendizaje.

Otra línea principal es seguir fortaleciendo la integración docente-asistencial- investigativa a los distintos niveles del Sistema Nacional de Salud (SNS), sobre todo en la atención primaria. Los distintos planos de integración forjados en estos años han posibilitado el nivel de desarrollo actual de la Educación Médica Superior, pero su ulterior perfeccionamiento se convierte en una premisa para alcanzar etapas superiores de desarrollo. 
Tabla 1. Programas de las disciplinas de Salud Pública y Formas de Organización de la Enseñanza. ISCMH 1994

\begin{tabular}{|c|c|c|c|c|c|c|c|c|}
\hline \multirow{3}{*}{ Asignatura } & \multicolumn{8}{|c|}{ FOE } \\
\hline & \multicolumn{2}{|c|}{$T$} & \multicolumn{2}{|c|}{$\mathrm{P}$} & \multicolumn{2}{|c|}{ ET } & \multicolumn{2}{|c|}{ Total } \\
\hline & No. & $\% *$ & No. & $\% *$ & No. & $\% *$ & No. & $\%=\%$ \\
\hline Filosofía y Salud & 116 & 100 & - & & - & & 116 & 8,6 \\
\hline $\begin{array}{l}\text { Bioestadística } \\
\text { y Computación }\end{array}$ & 18 & 28,1 & 46 & 71,9 & - & & 64 & 4,8 \\
\hline Psicología Medica & 56 & 63,6 & 32 & 36,4 & - & & 88 & 6,6 \\
\hline Sociedad y Salud & 34 & 30,4 & 78 & 69,6 & - & & 112 & 8,3 \\
\hline Higiene y Epidemiología & 42 & 28,4 & 60 & 40,5 & 46 & 31,0 & 148 & 11,0 \\
\hline Administración de Salud & 9 & 12,5 & 17 & 23,6 & 46 & 63,9 & 72 & 5,4 \\
\hline $\begin{array}{l}\text { Medicina General } \\
\text { Integral (MGI) }\end{array}$ & 37 & 5,0 & 6 & 0,8 & 699 & 94,2 & 742 & 55,3 \\
\hline Total & 312 & 23,2 & 239 & 17,8 & 791 & 59,0 & 1342 & 100,0 \\
\hline
\end{tabular}

* \% calculado en base al total de la fila, *s \% calculado en base al total de la columna, FOE: forma organización de la enseñanza, T: teoría, P: práctica,

ET: educación en el trabajo.

Fuente: plan de estudios 1985.

Tabla 2. Disciplinas de Salud Pública y formas de organización de la enseñanza en el plan de estudio perfeccionado en 2010

\begin{tabular}{|c|c|c|c|c|c|c|c|c|c|c|}
\hline \multirow{3}{*}{$\begin{array}{l}\text { Asignatura o } \\
\text { Disciplinas }\end{array}$} & \multicolumn{10}{|c|}{ FOE } \\
\hline & \multicolumn{2}{|c|}{$\mathrm{T}$} & \multicolumn{2}{|c|}{$\mathrm{CP}, \mathrm{S}, \mathrm{TI}$} & \multicolumn{2}{|c|}{ ET } & \multicolumn{2}{|c|}{ Eval } & \multicolumn{2}{|c|}{ Total } \\
\hline & No. & $\% *$ & No. & \%* & No. & \%* & No. & $\%$ & No. & $\% * *$ \\
\hline $\begin{array}{l}\text { Medicina } \\
\text { General } \\
\text { Integral (MGI) }\end{array}$ & 37 & 3,9 & 265 & 28,25 & 595 & 63,4 & 41 & 4,3 & 938 & 46,8 \\
\hline $\begin{array}{l}\text { Filosofia y } \\
\text { Sociedad }\end{array}$ & 40 & 35,7 & 72 & 64,2 & - & - & - & - & 112 & 5,6 \\
\hline $\begin{array}{l}\text { Informática } \\
\text { Médica }\end{array}$ & 25 & 20,8 & 83 & 69,0 & - & - & 12 & 10,0 & 120 & 6,0 \\
\hline $\begin{array}{l}\text { Psicología } \\
\text { Médica }\end{array}$ & 24 & 25,0 & 64 & 66,0 & - & - & 8 & 8,3 & 96 & 4,8 \\
\hline Salud Pública & 18 & 7,4 & 90 & 37,0 & 126 & 52,0 & 8 & 3,3 & 242 & 12,0 \\
\hline Sub Total & 144 & 9,5 & 574 & 37,9 & 721 & 47,6 & 69 & 4,5 & 1514 & - \\
\hline $\begin{array}{l}\text { Rotación } \\
\text { Internado de } \\
\text { MGI }\end{array}$ & - & - & - & - & 496 & 40,8 & - & - & 496 & 24,7 \\
\hline Total & 144 & 7,1 & 574 & 28,6 & 1217 & 60,7 & 69 & 3,4 & 2004 & 100,0 \\
\hline
\end{tabular}

* \% calculado en base al total de la fila, ** \% calculado en base al total de la columna, FOE: forma organización de la enseñanza, T: teoría, P: práctica, ET: educación en el trabajo, CP: clases prácticas, S: seminarios, TI: trabajo independiente, Eval: evaluación.

Fuente: plan de estudios 1985. 


\section{Las disciplinas de salud pública en el plan perfeccionado en el 2010}

Aquí se presentan las características de los programas de las asignaturas de Salud Pública en el plan de estudios de la carrera de medicina perfeccionado en el 2010.

En la tabla 2 se muestran en general las asignaturas y disciplinas que conforman las disciplinas de Salud Pública en el plan de estudio modificado y se evidencia que la disciplina de $\mathrm{MGI}$, que la conforman las cinco asignaturas de 1ro. a 5to. año más la rotación de MGI del internado, acumula 1434 h, la mayor cantidad del fondo de tiempo de este grupo, lo que significa el $71,5 \%$. También constituye la disciplina con mayor representación en el plan de estudio ya que utiliza el 13,2 \% de las 10 $862 \mathrm{~h}$ totales del plan.

En cuanto a las formas de organización de la enseñanza, el 60,7 \% se realiza sobre la base de la Educación en el Trabajo, aspecto que caracteriza a la formación del recurso humano en las ciencias de la salud en Cuba. Las Conferencias han disminuido al 7,1 \% de las horas lectivas del grupo de asignaturas y disciplinas de Salud Pública y los Seminarios, Clases prácticas y Trabajo independiente, se han elevado a $28,6 \%$. Estos cambios en las formas organizativas de la enseñanza, dando prioridad a aquellas donde el estudiante juega un papel activo, nos demuestra que la docencia se centra en que este tenga un papel cada vez más protagónico y se convierta en el sujeto de su propia educación (tabla 2).

\section{DISCUSIÓN}

\section{Análisis de los programas de las disciplinas de Salud Pública de la carrera de medicina. 1994 y 2010}

Los datos relacionados con el programa de 1994 se corresponden con los resultados de la investigación realizada por el ISCM H y la OPS.

Medicina General Integral. Una de las recomendaciones del estudio de 1994 fue que la disciplina de MGI se convirtiera en la asignatura integradora del plan de estudio de la carrera de medicina, lo cual se cumplió en el perfeccionamiento del plan de estudio ejecutado en el 2010. En el 1994 se impartían dos asignaturas de MGI y una rotación de siete semanas en el internado rotatorio. En el 2010 son cinco las asignaturas de MGI que se imparten, más el internado rotatorio. En el 1994 se hicieron $742 \mathrm{~h}$ de clases en las dos estancias y la rotación, lo que significa el $55 \%$ de las horas del grupo de las asignaturas de Salud Pública. En el 2010 se impartieron $1434 \mathrm{~h}$ en la disciplina MGI, lo que significa un incremento de $692 \mathrm{~h}$ lectivas en el plan para el $71 \%$ de las horas del grupo de asignaturas de Salud Pública y que representa un aumento de $93 \%$. Se reordenaron y perfeccionaron los contenidos de los programas de esta disciplina, que tributan al desarrollo de las funciones de promoción, prevención, recuperación y rehabilitación inherentes al médico formado con este perfil.

Filosofía y Sociedad. Comparando ambos programas se aprecia que el programa perfeccionado ha ganado en la diversificación de las formas de enseñanza. En el estudio que se realizó en el 1994, las 116 h de clases de esta disciplina eran de Conferencias. En el programa de la disciplina elaborado en el 2010, las Conferencias son solo $40 \mathrm{~h}$ y se imparten $38 \mathrm{~h}$ de Clases Prácticas y $34 \mathrm{~h}$ de Seminarios. Además, los estudiantes participan en jornadas científicas, cátedras 
multidisciplinarias, eventos de ética y bioética así como otras actividades extracurriculares que complementan la formación del estudiante.

Informática Médica. La comparación de esta disciplina en el plan de estudios perfeccionado de la carrera de medicina en el 2010, en relación con el estudio realizado en 1994, es positiva y consideramos que se han tomado en cuenta las recomendaciones de aquel estudio. Nos basamos en lo siguiente: el número de horas de docencia prácticamente se duplica. En el programa de 1994 se impartían $64 \mathrm{~h}$ de docencia y en la actualidad es de 120 , sin contar la evaluación. Este aumento en lo fundamental es en base a Clases Prácticas y la introducción de $8 \mathrm{~h}$ de Seminarios, forma de la enseñanza que en el programa de 1994 no se utilizaba.

De una sola asignatura que existía en 1994, Ilamada Bioestadística y Computación, hoy son dos asignaturas, la Informática que se imparte en el ler. año y la Metodología de la Investigación y Estadísticas que se imparte en el segundo. Su ubicación por semestre de la carrera se realiza dependiendo de las condiciones profesorales de cada centro de educación médica superior (CEMS).

Uno de los aspectos evaluados en 1994 era que los conocimientos de Informática y de Metodología de la Investigación y Estadísticas no debían ser objeto solamente de una o dos asignaturas, sino que debían estar presentes en todas. En el perfeccionamiento del plan de estudios de la carrera está presente, como hecho novedoso, la formulación de la Estrategia Curricular de Investigación e Informática. ${ }^{2}$

Psicología Médica. Con el programa estudiado en 1994 los educandos adquirían la dimensión psicológica del proceso salud-enfermedad y de la atención de salud, fundamentalmente dirigido a la atención a pacientes ingresados, que entraña un enfoque que se centra en el individuo, la enfermedad, la curación y el hospital. Uno de los señalamientos efectuados en 1994 a esta disciplina fue que en la asignatura Psicología Médica I, ${ }^{3}$ no se realizaban acciones de Educación en el Trabajo, lo que persiste en el plan perfeccionado. En el programa de Psicología Médica II, ${ }^{4}$ tampoco tiene concebido esta forma de enseñanza. Esto nos hace pensar que otra de las valoraciones de aquel estudio de 1994 que señalaba debía vincularse más a la APS, sigue igual. Aunque en las clases se destaca con importancia este nivel de atención a la salud, al no contar con actividades de Educación en el Trabajo en la APS, no se adquieren las habilidades ni se consolidan los conocimientos al no ejercitarlos en la práctica. Es cierto que en MGI se retoman algunos contenidos de los programas de Psicología a través de la Educación en el Trabajo de esta disciplina, más sigue siendo válida la recomendación de 1994, por lo que estimamos que en Psicología Médica, las consideraciones realizadas en el estudio en 1994 mantienen su vigencia.

Salud Pública. En el plan de estudios analizado en 1994, la asignatura estancia se impartía a tiempo completo en los policlínicos y los consultorios médicos de familia (CMF), así como en los centros municipales y provinciales de Higiene y

Epidemiología y se introdujo la forma organizativa de la enseñanza de Educación en el Trabajo. No obstante estos avances, no se lograba una visión integral del alumno de los problemas de salud pública de un sector de salud, poco dominio de las medidas de promoción y prevención, de las estadísticas sanitarias, de la influencia del medio ambiente así como la modificación de los estilos y modos de vida saludables de la población y no se articulaba bien el Análisis de la Situación de Salud (ASIS) sobre todo con muchas deficiencias en la elaboración de los Planes de Acción y de Ejecución. En al análisis de 1994 se concluyó que "resulta necesario seguir abordando el perfeccionamiento de este programa y la organización de todo el bloque de asignaturas médico-sociales del 5 to. año de la carrera de medicina". ${ }^{1}$ En 1996 se unificaron las asignaturas- estancias de teoría y administración de salud (TAS) y de higiene y epidemiología bajo el nombre de Salud Pública. 
En abril de 2010 se perfeccionó este programa añadiendo y suprimiendo temas y perfeccionando su unidad interna. En la nueva estancia integrada y según la experiencia del autor, se tiene la impresión que el alumno desarrolla más habilidades que cuando se daba por separado TAS e Higiene y Epidemiología en 1994, se apropia con mayor facilidad de los conceptos básicos de la Salud Pública y puede articular sus conocimientos con mayor precisión. Aunque ya en 5to. año ya el alumno cursó asignaturas relacionadas con la Salud Pública, aún no domina los principales problemas de salud de la población cubana, las estadísticas demográficas, de morbilidad, mortalidad, los componentes y determinantes del Estado de Salud de la Población, los indicadores de salud y la influencia tan marcada del ambiente en los problemas de salud de la comunidad. En esta asignatura, el estudiante se apropia del método epidemiológico y domina con mayor claridad la conducta a seguir en el control y seguimiento de las enfermedades transmisibles y no transmisibles y desarrolla habilidades de vigilancia en salud.

También en esta estancia se provee al estudiante de los objetivos y prioridades del sistema de salud para el año en cuestión, el reordenamiento del sistema de salud, la reorganización, redimensionamiento y compactación de los servicios, los propósitos del Ministerio de Salud Pública para el año y su perspectiva hasta el 2015. Se le educa en que todas las medidas que se están tomando en Salud Pública y que ellos ven reflejado en su policlínico persiguen los propósitos de hacerla más eficiente y eficaz, sostenible, que coadyuve a incrementar la satisfacción de la población y mejorar los indicadores de salud.

Por lo general, el ASIS que realiza el estudiante, es de mayor calidad que el ejecutado por el médico de familia, cuando lo tiene, ya que nos hemos encontrado consultorios donde no lo han confeccionado. En muchos de ellos se presentan los siguientes problemas: no participan en su confección los factores de la zona, las organizaciones políticas y de masas, no se discute con la población los problemas de salud y la conducta a seguir ante ellos, o sea, no hay participación popular, los planes de acción y los planes de ejecución están mal confeccionados, no se priorizan los problemas de salud más importantes, los datos de la población que atiende están incompletos y otras irregularidades.

Analizando la estancia actual con la que se hacía en 1994, estimamos que esta es de mayor calidad y elimina las limitaciones halladas en el estudio de 1994.

La Estrategia Curricular de Salud Pública y Formación Ambiental ${ }^{5}$ es un tema totalmente nuevo en el plan de estudios de la carrera de medicina y se debe al perfeccionamiento que se realizó en el 2010, donde se fundamenta en los objetivos terminales del médico a formar elaborados por la Comisión Nacional de Carrera en el documento "Estrategia para el Perfeccionamiento del Plan de Estudios de la carrera de medicina."

\section{Comparación de ambos planes}

Existen entre ambos planes de estudio diferencias cuantitativas y cualitativas en las asignaturas de Salud Pública que gana espacios en el plan perfeccionado, nueva nominación de algunas y contenidos de mayor pertinencia. Los objetivos poseen un mayor alcance y profundidad, y las formas de organización de la enseñanza se amplían sensiblemente la educación en el trabajo y las clases prácticas.

La asignatura Sociedad y Salud del plan de estudios de 1985, después de dos transformaciones, se convirtió en Introducción a la Medicina General Integral, ampliando los objetivos y contenidos que tenía esta. La asignatura de Bioestadística 
y Computación se convirtió en la disciplina de Informática Médica constituida por dos asignaturas, Informática y Metodología de la Investigación y Estadística. Las asignaturas de Higiene y Epidemiología y la Teoría de Administración de Salud se unieron y formaron la asignatura Salud Pública. La disciplina de Medicina General Integral, que tenía dos asignaturas y una rotación de siete semanas en el internado, se ha convertido en una disciplina integradora de cinco asignaturas, Introducción a la MGI, Promoción de Salud, Prevención en Salud, Medicina Comunitaria y MGI propiamente dicha, persistiendo la rotación del internado. Se mantienen las dos asignaturas de Psicología y las dos de Filosofía.

En total son 12 asignaturas y una rotación del internado de siete semanas para 13 unidades curriculares con un total de $2004 \mathrm{~h}$, que si sumamos $160 \mathrm{~h}$ de dos tiempos electivos se eleva a $2164 \mathrm{~h}$ y 15 unidades curriculares de un plan de estudios de $10862 \mathrm{~h}$ lo que representa el $20 \%$ de las horas del plan de estudios.

Las horas lectivas de las disciplinas de Salud Pública en el plan de estudios de 1994 eran de $1342 \mathrm{~h}$ y en el plan perfeccionado asciende a 2164 , lo que significa un incremento de 822 h. Más aún, su distribución por formas organizativas de la enseñanza resulta superior. Se han reducido sustancialmente las Conferencias, de $312 \mathrm{~h}$ a 178, lo que significa 134 h menos para una disminución de $43 \%$. Las horas de Clases prácticas y Seminarios han aumentado de 239 a 546, lo que significa 307 h más para el 128 \%. La Educación en el Trabajo también tiene en el plan perfeccionado un incremento de $332 \mathrm{~h}$ ya que de $791 \mathrm{~h}$ pasa a 1217 para una ampliación de $41 \%$.

En el análisis particular que hemos hecho de cada disciplina y asignatura se ha demostrado que en todas ha habido cierto grado de mejora y en algunas de gran magnitud, a excepción de Psicología Médica, donde se mantienen las recomendaciones del informe de 1994.

El porcentaje de las horas de las asignaturas de Salud Pública se elevó al $20 \%$ de las horas totales del plan de estudio. En el plan analizado en 1994 solo era de $17 \%$. En el análisis que realizamos del plan de estudios de 1994, las disciplinas de Salud Pública y sus asignaturas se abordaban tardíamente sin coordinación horizontal ni vertical.

El plan perfeccionado contempla, entre otras, dos Estrategias Curriculares, una de Salud Pública y Formación Ambiental y otra de Estrategia Curricular de Investigaciones e informática, que permean todas las unidades curriculares de la carrera. También está presente la Estrategia Curricular de Medicina Natural y Tradicional que tiene puntos de contacto con la Salud Pública (tabla 3).

En general se puede señalar que las limitaciones identificadas en la investigación de 1994 del ISCM H y OPS, han sido eliminadas en el perfeccionamiento del plan de estudio del 2010 en consonancia con las consideraciones de este estudio, destacándose la MGI como la disciplina integradora de todo el currículo.

Se incrementaron las horas lectivas de los programas de las disciplinas de Salud Pública con respecto al total de horas del plan de estudio, de $17 \%$ en 1994 a $20 \%$ en el 2010. Se han mejorado sus formas organizativas de enseñanza, privilegiando la Educación en el Trabajo, las Actividades prácticas y Seminarios y la Actividad independiente del estudiante. 
Tabla 3. Distribución de horas por años y totales según unidades curriculares y contenidos de salud pública ${ }^{63}$

\begin{tabular}{|c|c|c|c|}
\hline Años y sus horas & $\begin{array}{c}\text { Unidades } \\
\text { curriculares (*) }\end{array}$ & $\begin{array}{l}\text { Horas } \\
\text { totales }\end{array}$ & Porcentaje \\
\hline $1^{0} 1329$ & 5 & 617 & 46,43 \\
\hline $2^{\circ} 1392$ & $\begin{array}{l}4 \\
5 * *\end{array}$ & $\begin{array}{l}451 \\
531 * * *\end{array}$ & $\begin{array}{l}32,40 \\
38,15\end{array}$ \\
\hline $3^{\circ} 1895$ & 1 & $44 * * * *$ & 2,32 \\
\hline $4^{\circ} 1960$ & $\begin{array}{l}0 \\
1 * *\end{array}$ & $\begin{array}{l}0 \\
80 * * *\end{array}$ & $\begin{array}{l}0,0 \\
4,08\end{array}$ \\
\hline $5^{\circ} 1502$ & 2 & 402 & 26,76 \\
\hline Sub total 8078 & $\begin{array}{l}12 \\
14^{* *}\end{array}$ & $\begin{array}{l}1514 \\
1674\end{array}$ & $\begin{array}{l}18,74 \\
20,72\end{array}$ \\
\hline $\begin{array}{l}\text { Internado rotatorio } \\
2784\end{array}$ & 1 & $496 * * * * *$ & 17,82 \\
\hline $\begin{array}{l}\text { Total } \\
10862\end{array}$ & $\begin{array}{l}13 \\
15 * *\end{array}$ & $\begin{array}{l}2004 \\
2164\end{array}$ & $\begin{array}{l}18,50 \\
20,0\end{array}$ \\
\hline
\end{tabular}

(*) Incluye las unidades curriculares: Introducción a la MGI, Promoción de Salud, Prevención en Salud, Medicina Comunitaria, Medicina General Integral, Salud Pública, Informática Médica I, Metodología de la Investigación y Estadística, Psicología Médica I y II, Filosofía y Sociedad I y II y la rotación de MGI en el internado.

** Asciende las unidades curriculares sumando el tiempo electivo como de contenido de Salud Pública. *** Se incluyen $80 \mathrm{~h}$ de tiempo electivo.

**** No se incluyen horas de tiempo electivo como de contenidos sociomédicos. ***** Se sumaron $80 \mathrm{~h}$ del período evaluativo a la rotación MGI.

La utilización del nuevo concepto de las Estrategias Curriculares en la conformación del plan de estudios de la carrera de medicina y entre ellas la de Salud Pública y Formación Ambiental y la de Investigación e Informática, aportan una mayor coherencia vertical al currículo que debe contribuir a un logro más acabado de sus objetivos.

\section{REFERENCI AS BI BLI OGRÁFICAS}

1. Vela Valdés J. La enseñanza de las disciplinas de Salud Pública en el Plan de Estudios de Medicina. Situación actual y perspectivas. Washington, D.C.: OPS; 1994.

2. Comisión Nacional de Carrera de Medicina. Programa de la disciplina Filosofía y Sociedad. Abril 2010. La Habana: MINSAP; 2010.

3. _. Programa de la asignatura Psicología Médica I. Enero 2010. La Habana: MI N̄SAP'; 2010.

4. $20 \overline{1} 0$. . Programa de la asignatura Salud Pública. Abril 2010. La Habana: MINSAP; 
5. ____. Estrategia Curricular de Salud Pública y Formación Ambiental. Abril 2010. La Habana: MI NSAP; 2010.

6. de medicina. Objetivos terminales del médico a formar. La Habana: MINSAP; 2010.

Recibido: 6 de diciembre de 2011.

Aprobado: 31 de enero de 2012.

Juan Vela Valdés. Escuela Nacional de Salud Pública. Calle 100 No. 1132 e/ E y Perla. Altahabana, Municipio Boyeros. La Habana, Cuba. Correo electrónico: jvela@infomed.sld.cu, sacasas@infomed.sld.cu 